\title{
On an Anti-Ramsey Problem of Burr, Erdős, Graham, and T. Sós
}

\author{
Gábor N. Sárközy ${ }^{1,2}$ and Stanley Selkow ${ }^{\mathbf{1}}$ \\ ${ }^{1}$ COMPUTER SCIENCE DEPARTMENT \\ WORCESTER POLYTECHNIC INSTITUTE \\ 100 INSTITUTE ROAD \\ WORCESTER, MA 01609 \\ E-mail: gsarkozy@cs.wpi.edu \\ E-mail:sms@cs.wpi.edu \\ ${ }^{2}$ COMPUTER AND AUTOMATION RESEARCH INSTITUTE \\ HUNGARIAN ACADEMY OF SCIENCES \\ BUDAPEST, P.O. BOX 63 \\ BUDAPEST H-1518, HUNGARY
}

Received February 5, 2004; Revised October 10, 2005

Published online 25 January 2006 in Wiley InterScience(www.interscience.wiley.com).

DOI 10.2002/jgt.20148

Abstract: Given a graph $L$, in this article we investigate the anti-Ramsey number $\chi_{S}(n, e, L)$, defined to be the minimum number of colors needed to edge-color some graph $G(n, e)$ with $n$ vertices and e edges so that in every copy of $L$ in $G$ all edges have different colors. We call such a copy of $L$ totally multicolored (TMC).

In [7] among many other interesting results and problems, Burr, Erdős, Graham, and T. Sós asked the following question: Let $L$ be a connected bipartite graph which is not a star. Is it true then that

$$
\chi_{S}\left(n, \alpha n^{2}, L\right) / n \rightarrow \infty \quad \text { as } n \rightarrow \infty \text { ? }
$$

Contract grant sponsor: National Science Foundation (in part); Contract grant number: DMS-0456401.

(C) 2006 Wiley Periodicals, Inc. 
In this article, we prove a slightly weaker statement, namely we show that the statement is true if $L$ is a connected bipartite graph, which is not a complete bipartite graph. @ 2006 Wiley Periodicals, Inc. J Graph Theory 52: 147-156, 2006

Keywords: regularity lemma; anti-Ramsey number

\section{INTRODUCTION}

\section{A. Notation and Definitions}

We let $V(G)$ and $E(G)$ denote the vertex-set and the edge-set of the graph $G$, and $(A, B)$ or $(A, B, E)$ denote a bipartite graph $G=(V, E)$, where $V=A \cup B$, and $E \subset A \times B$. In general, given any graph $G$ and two disjoint subsets $A, B$ of $V(G)$, the pair $(A, B)$ is the graph restricted to $A \times B . N(v)$ is the set of neighbors of $v \in V$. Hence the size of $N(v)$ is $|N(v)|=\operatorname{deg}(v)=\operatorname{deg}_{G}(v)$, the degree of $v$. For a vertex $v \in V$ and set $U \subset V-\{v\}$, we denote by $N_{G}(v, U)$ the neighbors of $v$ in $U, \operatorname{deg}_{G}(v, U)=\left|N_{G}(v, U)\right| . P_{4}$ denotes the path with 4 vertices. We denote by $e(A, B)$ the number of edges of $G$ with one endpoint in $A$ and the other in $B$. For non-empty $A$ and $B$,

$$
d(A, B)=\frac{e(A, B)}{|A||B|}
$$

is the density of the graph between $A$ and $B$.

Definition 1. The pair $(A, B)$ is $\varepsilon$-regular if

$$
X \subset A, Y \subset B,|X|>\varepsilon|A|,|Y|>\varepsilon|B|
$$

imply

$$
|d(X, Y)-d(A, B)|<\varepsilon,
$$

otherwise it is $\varepsilon$-irregular.

A graph $L$ is embeddable into another graph $G$, if $G$ has a subgraph isomorphic to $L$, that is, if there is a one-to-one map (injection) $\phi: V(L) \rightarrow V(G)$ such that $(x, y) \in E(L)$ implies $(\phi(x), \phi(y)) \in E(G)$. Note that in this article subgraph does not mean an induced subgraph. In a graph two edges are called strongly independent, if they are disjoint and all four vertices span no other edge.

\section{B. Anti-Ramsey Problems}

In traditional Ramsey theory (see e.g. [9]) a typical question is the following. Given a graph $L$ and an integer $r>0$, which graphs $G$ have the property that, no matter 
how the edges of $G$ are $r$-colored, there is a monochromatic copy of $L$, in other words a subgraph of $G$ isomorphic to $L$ in which all edges have the same color.

In anti-Ramsey theory, we go in the opposite direction and we try to find edgecolorings such that all copies of $L$ have all edges of different colors. We call such a copy of $L$ totally multicolored (TMC). Anti-Ramsey numbers were introduced by Erdős, Simonovits, and Sós [8] in the 1970s, and they have been actively studied recently (see e.g. $[1-4,6,7,13]$ ).

The following extremal anti-Ramsey numbers were introduced and studied in [6] and [7] (see also [10] and [11]). Define $\chi_{S}(n, e, L)$ to be the smallest integer $r$ such that there exists a graph $G$ with $n$ vertices and $e$ edges that has an edge-coloring in $r$ colors such that every $L$ in $G$ is TMC. This notation comes from the fact that the value we seek is also the strong chromatic number of the hypergraph which has as its set of points the edges of $G$, with (hyper)edges consisting of the sets of edges of $G$ which form copies of $L$. It turns out that the determination of $\chi_{S}(n, e, L)$ is surprisingly deep. It is closely related, for example, to the celebrated function $r_{k}(n)$, the size of the largest subset of $\{1,2, \ldots, n\}$ containing no $k$-term arithmetic progression. Among many other interesting results and problems, in [6] and [7] the authors proved the following result for bipartite $L$ 's which contain two strongly independent edges.

Theorem 1 ([16]). Suppose L is a bipartite graph having at least two strongly independent edges, and maximum degree at least two. Then for any $\alpha>0$, if $e>$ $\alpha n^{2}$, we have

$$
\chi_{S}(n, e, L)>\alpha^{\prime} n^{2}
$$

for some fixed positive constant $\alpha^{\prime}$ depending on $\alpha$.

On the other hand, whenever $L$ does not have two strongly independent edges (even when $L$ is not necessarily bipartite), $\chi_{S}(n, e, L)$ can be much smaller.

Theorem 2 ([6]). If no two edges of L are strongly independent and $<<\left(\frac{1}{2}-\varepsilon\right) n^{2}$ for some fixed $\varepsilon>0$, then

$$
\chi_{S}(n, e, L)=O\left(n^{2} / \log n\right)
$$

For the special case $L=P_{4}$, even stronger results were obtained.

Theorem 3 ([7]). We have

$$
\chi_{S}\left(n, \operatorname{cnr}_{3}(n), P_{4}\right) \leq n \text { for a suitable c }>0 .
$$

For any $\alpha>0$, we have $\chi_{S}\left(n, \alpha n^{2}, P_{4}\right)>$ cn for any $c$ if $n$ is sufficiently large. 
With this last result in mind, in [7] the authors asked the following question. Let $L$ be a connected bipartite graph which is not a star. Is it true that

$$
\chi_{S}\left(n, \alpha n^{2}, L\right) / n \rightarrow \infty \text { as } n \rightarrow \infty \text { ? }
$$

Note that the statement is clearly not true for stars. In this article, we prove a slightly weaker statement. Namely we show that the statement is true if $L$ is a connected bipartite graph which is not a complete bipartite graph.

Theorem 4. For any $\alpha, c>0$ there exists an $n_{0}=n_{0}(\alpha, c)$ such that if $n \geq n_{0}$, $e>\alpha n^{2}$ and $L$ is a connected bipartite graph which is not a complete bipartite graph, then

$$
\chi_{S}(n, e, L)>c n \text {. }
$$

However, the original question still remains open for complete bipartite graphs that are not stars, for instance for $C_{4}$.

In the next section, we provide the tools, including the Regularity Lemma. Then in Section 3 we prove the theorem by essentially reducing the general case to that of $P_{4}$.

\section{TOOLS}

In the proof the Regularity Lemma of Szemerédi [12] plays a central role. Here we will use the following variation of the lemma.

Lemma 1 (Regularity Lemma - Degree form). For every $\varepsilon>0$ there is an $M=M(\varepsilon)$ such that if $G=(V, E)$ is any graph and $d \in[0,1]$ is any real number, then there is a partition of the vertex-set $V$ into $t+1$ sets (so-called clusters) $C_{0}, C_{1}, \ldots, C_{t}$, and there is a subgraph $G^{\prime}=\left(V, E^{\prime}\right)$ with the following properties:

- $t \leq M$,

- $\left|C_{0}\right| \leq \varepsilon|V|$,

- all clusters $C_{i}, i \geq 1$, are of the same size,

- $\operatorname{deg}_{G^{\prime}}(v)>\operatorname{deg}_{G}(v)-(d+\varepsilon)|V|$ for all $v \in V$,

- $\left.G^{\prime}\right|_{C_{i}}=\emptyset i \geq 1,\left(C_{i}\right.$ are independent in $\left.G^{\prime}\right)$,

- all pairs $\left.G^{\prime}\right|_{C_{i} \times C_{j}}, 1 \leq i<j \leq t$, are $\varepsilon$-regular, each with density 0 or exceeding $d$.

This form (see [10]) can easily be obtained by applying the original Regularity Lemma (with a smaller value of $\varepsilon$ ), adding to the exceptional set $C_{0}$ all clusters incident to many irregular pairs, and then deleting all edges between any other clusters where the edges either do not form a regular pair or they do but with a density at most $d$. 
We will also use a simple lemma from [6].

Lemma 2. Let $X$ be a set of $N$ elements, $0<\beta<1, k=\left\lceil\frac{2}{\beta}\right\rceil$. Suppose that $B_{1}, \ldots, B_{k} \subset X$ satisfy $\left|B_{i}\right| \geq \beta N$. Then there exist $1 \leq i<j \leq k$ with

$$
\left|B_{i} \cap B_{j}\right|>\frac{\beta^{2} N}{5} .
$$

Proof. Suppose the contrary and let us bound the size of the union of the sets.

$$
\begin{aligned}
N & \geq\left|B_{1} \cup \ldots \cup B_{k}\right| \geq \sum_{i=1}^{k}\left|B_{i}\right|-\sum_{1 \leq i<j \leq k}\left|B_{i} \cap B_{j}\right| \geq k \beta N-\left(\begin{array}{l}
k \\
2
\end{array}\right) \frac{\beta^{2} N}{5} \\
& >2 N-\frac{9}{10} N>N,
\end{aligned}
$$

which is a contradiction.

\section{PROOF OF THEOREM 4}

Let $c, \alpha>0$ be fixed real numbers; assume that $c \geq 1$ without loss of generality. Write $|V(L)|=l$. Suppose that $G$ is a graph with $n$ vertices and $e$ edges where $e>\alpha n^{2}$ and $n$ is sufficiently large. Suppose further that the edges of $G$ are colored by at most $c n$ colors. We will show that there is a copy of $L$ in $G$ that is not TMC, implying the theorem. Thus we want to find an embedding $\phi: V(L) \rightarrow V(G)$ such that the embedded copy of $L$ is not TMC.

Let us denote the two color classes in the bipartition of $L$ by $A$ and $B$. Since $L$ is not a complete bipartite graph, it contains a vertex from $A$ and a vertex from $B$ which are not adjacent. Furthermore, since $L$ is connected there is an edge leaving both of these vertices. Finally, the other endpoints of these two edges must be connected by an edge because otherwise we have two strongly independent edges in $L$, and then a much stronger result is true than Theorem 4 (see Theorem 1). Thus by the above, there is an induced $P_{4}$ in $L$, which we denote by $\left(a_{1}, b_{1}, a_{2}, b_{2}\right)$ where $a_{i} \in A, b_{i} \in B, i=1,2$. Denote the remaining vertices of $L$ (if there are any) in $A$ by $a_{3}, \ldots, a_{|A|}$ and in $B$ by $b_{3}, \ldots, b_{|B|}$. First we will find an embedding of this $P_{4}$ with a repeated color, then an arbitrary embedding of the rest of $L$, thus giving a copy of $L$ in $G$ that is not TMC.

Let us apply the degree form of the Regularity Lemma (Lemma 1 ) for $G$ with

$$
d=\frac{\alpha}{2} \quad \text { and } \quad \varepsilon=\left(\frac{\alpha}{14 c}\right)^{15 l} .
$$


Denote

$$
N=\left|C_{1}\right|=\ldots=\left|C_{t}\right|=\frac{n-\left|C_{0}\right|}{t} .
$$

Let us remove first from $G^{\prime}$ the vertices of $C_{0}$ and all the edges incident to them. Then we remove all exceptional edges $(u, v)$ for which if $u \in C_{i}, v \in C_{j}, i, j \geq 1$ then either we have

$$
\operatorname{deg}_{G^{\prime}}\left(u, C_{j}\right)<(d-\varepsilon) N=\left(\frac{\alpha}{2}-\varepsilon\right) N,
$$

or

$$
\operatorname{deg}_{G^{\prime}}\left(v, C_{i}\right)<(d-\varepsilon) N=\left(\frac{\alpha}{2}-\varepsilon\right) N
$$

Let us denote the resulting graph by $G^{\prime \prime}$.

By the $\varepsilon$-regularity of the pair $\left.G^{\prime}\right|_{C_{i} \times C_{j}}$ with density exceeding $d$, at most $2 \varepsilon N^{2}$ exceptional edges of the pair $\left.G^{\prime}\right|_{C_{i} \times C_{j}}$ are removed, and thus at most $2 \varepsilon n^{2}$ exceptional edges of $G^{\prime}$ are removed in total which are not adjacent to $C_{0}$.

Then using (1) we have

$$
\begin{aligned}
\left|E\left(G^{\prime \prime}\right)\right| & =\frac{1}{2} \sum_{v \in V\left(G^{\prime \prime}\right)} \operatorname{deg}_{G^{\prime \prime}}(v) \geq \frac{1}{2} \sum_{v \in V\left(G^{\prime \prime}\right)}\left(\operatorname{deg}_{G^{\prime}}(v)-\left|C_{0}\right|\right)-2 \varepsilon n^{2} \\
& >\frac{1}{2} \sum_{v \in V\left(G^{\prime \prime}\right)}\left(\operatorname{deg}_{G}(v)-(d+\varepsilon) n-\left|C_{0}\right|\right)-2 \varepsilon n^{2} \\
& \geq \frac{1}{2} \sum_{v \in V\left(G^{\prime \prime}\right)} \operatorname{deg}_{G}(v)-\frac{d+6 \varepsilon}{2} n^{2} \\
& =\frac{1}{2} \sum_{v \in V(G)} \operatorname{deg}_{G}(v)-\frac{1}{2} \sum_{v \in C_{0}} \operatorname{deg}_{G}(v)-\frac{d+6 \varepsilon}{2} n^{2} \\
& \geq|E(G)|-\frac{d+7 \varepsilon}{2} n^{2} \geq \frac{\alpha}{2} n^{2} .
\end{aligned}
$$

Furthermore, in $G^{\prime \prime}$ for every pair $\left.G^{\prime \prime}\right|_{C_{i} \times C_{j}}$ and $X \subset C_{i}, Y \subset C_{j},|X|,|Y| \geq$ $(\varepsilon)^{1 / 3} N$, if there is an edge between $C_{i}$ and $C_{j}$ in $G^{\prime}$ (and so the density exceeds $d$ ), then by (1) and the $\varepsilon$-regularity of the pair $\left.G^{\prime}\right|_{C_{i} \times C_{j}}$ we have

$$
d_{G^{\prime \prime}}(X, Y) \geq d_{G^{\prime}}(X, Y)-\frac{2 \varepsilon N^{2}}{|X||Y|} \geq d-\varepsilon-2(\varepsilon)^{1 / 3}>\frac{\alpha}{3} .
$$


Finally, for any $u \in C_{i}$ and $C_{j}$

$$
\operatorname{deg}_{G^{\prime}}\left(u, C_{j}\right) \geq(d-\varepsilon) N \quad \text { implies } \quad \operatorname{deg}_{G^{\prime \prime}}\left(u, C_{j}\right) \geq(d-2 \varepsilon) N>\frac{\alpha}{3} N .
$$

In the remainder of the proof we will first distinguish two cases. In both cases we will first embed the $P_{4}=\left(a_{1}, b_{1}, a_{2}, b_{2}\right)$ in $L$ in such a way that the embedded edges $\left(\phi\left(a_{1}\right), \phi\left(b_{1}\right)\right),\left(\phi\left(b_{1}\right), \phi\left(a_{2}\right)\right)$, and $\left(\phi\left(a_{2}\right), \phi\left(b_{2}\right)\right)$ will contain a repeated color and for some pair $\left(C_{i}, C_{j}\right)$ we will have

$$
\left|N_{G^{\prime \prime}}\left(\phi\left(a_{1}\right), C_{j}\right) \cap N_{G^{\prime \prime}}\left(\phi\left(a_{2}\right), C_{j}\right)\right| \geq \frac{1}{5}\left(\frac{\alpha}{3}\right)^{2} N,
$$

and

$$
\left|N_{G^{\prime \prime}}\left(\phi\left(b_{1}\right), C_{i}\right) \cap N_{G^{\prime \prime}}\left(\phi\left(b_{2}\right), C_{i}\right)\right| \geq \frac{1}{5}\left(\frac{\alpha}{3}\right)^{2} N .
$$

(5) and (6) guarantee that we will be able to finish the embedding of the rest of $L$, and thus we will get a copy of $L$ in $G^{\prime \prime}$ (and thus in $G$ ) that is not TMC.

Case 1. There is a color class (say red) in $G^{\prime \prime}$ which contains a star with at least $k=\left\lceil\frac{6}{\alpha}\right\rceil$ leaves. Let us consider this red star and denote the middle vertex by $u$ and the leaves by $v_{1}, v_{2}, \ldots, v_{k}$. Assume that $u \in C_{j}$. Apply Lemma 2 with $X=C_{j}$, $B_{i}=N_{G^{\prime \prime}}\left(v_{i}, C_{j}\right)$, and $\beta=\frac{\alpha}{3}$. By (4) we have in fact

$$
\left|B_{i}\right| \geq \frac{\alpha}{3} N \quad \text { for every } \quad 1 \leq i \leq k .
$$

Then we get two $B_{i}$ 's, say for simplicity $B_{1}$ and $B_{2}$, such that

$$
\left|B_{1} \cap B_{2}\right| \geq \frac{1}{5}\left(\frac{\alpha}{3}\right)^{2} N .
$$

Assume that $v_{1} \in C_{i}$. Let us embed $P_{4}$ in this case in the following way:

$$
\phi\left(a_{1}\right)=v_{1}, \phi\left(b_{1}\right)=u, \phi\left(a_{2}\right)=v_{2},
$$

and let $\phi\left(b_{2}\right)$ be a vertex $w$ in $B_{1} \cap B_{2}$ for which we have

$$
\left|N_{G^{\prime \prime}}\left(u, C_{i}\right) \cap N_{G^{\prime \prime}}\left(w, C_{i}\right)\right| \geq\left(\frac{\alpha}{3}\right)^{2} N .
$$

Using (3) with $X=\left(B_{1} \cap B_{2}\right) \backslash\{u\}$ and $Y=N_{G^{\prime \prime}}\left(u, C_{i}\right)$ guarantees that such a $w$ can be chosen.

The above embedding of $P_{4}$ has a repeated color (red) and (7) and (8) imply that it satisfies (5) and (6). Note that here actually we get somewhat more; the endpoints 
of $P_{4}$ are also connected in the embedding, so in this case we can embed $L$ even if it is a complete bipartite graph.

Case 2. There is no monochromatic star with at least $\left\lceil\frac{6}{\alpha}\right\rceil$ leaves.

However, this and (2) imply that in this case there is a monochromatic (say blue) matching $M$ in $G^{\prime \prime}$ such that

$$
|M| \geq \frac{1}{\left\lceil\frac{6}{\alpha}\right\rceil} \frac{\frac{\alpha}{2} n^{2}}{c n}>\frac{\alpha^{2}}{14 c} n .
$$

Write $U=V(M)$ for the vertex set of $M$. (9) implies that

$$
|U|>\frac{\alpha^{2}}{7 c} n
$$

Write also $U_{i}=U \cap C_{i}$. Define $I=\left\{i|| U_{i} \mid>\frac{\alpha^{2}}{14 c} N\right\}$, and set $U^{\prime}=\cup_{i \in I} U_{i}$ and $U^{\prime \prime}=U \backslash U^{\prime}$. Clearly $\left|U^{\prime \prime}\right| \leq \frac{\alpha^{2}}{14 c} n$. Since $|U|>\frac{\alpha^{2}}{7 c} n$, we have two vertices $y, z \in U^{\prime}$ adjacent in $M$. Let $y \in C_{i}$ and $z \in C_{j}$. In $G^{\prime \prime}$, we have at least one edge between $C_{i}$ and $C_{j}$, and hence by (3) we have

$$
d_{G^{\prime \prime}}\left(U_{i}, U_{j}\right)>\frac{\alpha}{3}
$$

We will remove some additional exceptional edges from $\left.G^{\prime \prime}\right|_{U_{i} \times U_{j}}$ and then we embed $L$ in the remaining bipartite graph. For a vertex $u$ covered by $M$, let us denote its pair in $M$ by $u^{\prime}$. We will remove all exceptional edges $(u, v)$ from $\left.G^{\prime \prime}\right|_{U_{i} \times U_{j}}$ for which if $u \in C_{i}, v \in C_{j}$, then either we have

$$
\left|N_{G^{\prime \prime}}\left(u, C_{j}\right) \cap N_{G^{\prime \prime}}\left(v^{\prime}, C_{j}\right)\right|<\left(\frac{\alpha}{3}\right)^{2} N,
$$

or

$$
\left|N_{G^{\prime \prime}}\left(v, C_{i}\right) \cap N_{G^{\prime \prime}}\left(u^{\prime}, C_{i}\right)\right|<\left(\frac{\alpha}{3}\right)^{2} N .
$$

Note that if $(u, v)$ is an edge in $M$ then $(u, v)$ is automatically an exceptional edge. For a fixed $u \in C_{i}$, the number of exceptional edges violating (12) incident to $u$ is at most $\varepsilon^{1 / 3} N$, since otherwise we get a contradiction with (3) by choosing $X=N_{G^{\prime \prime}}\left(u^{\prime}, C_{i}\right)$ and $Y$ to be the other endpoints of the exceptional edges. Thus the total number of exceptional edges violating (12) is at most $\varepsilon^{1 / 3} N^{2}$. Similarly, the total number of exceptional edges violating (11) is at most $\varepsilon^{1 / 3} N^{2}$. 
By (1) and (10), if we remove the exceptional edges, we still have more than

$$
\frac{\alpha}{3}\left|U_{i}\right|\left|U_{j}\right|-2 \varepsilon^{1 / 3} N^{2} \geq\left(\frac{\alpha}{3}\left(\frac{\alpha^{2}}{14 c}\right)^{2}-2 \varepsilon^{1 / 3}\right) N^{2}>0
$$

edges in $\left.G^{\prime \prime}\right|_{U_{i} \times U_{j}}$.

Let us take one such non-exceptional edge $(u, v)$, then it does not belong to $M$, and we embed $\phi\left(a_{1}\right)=v^{\prime}, \phi\left(b_{1}\right)=v, \phi\left(a_{2}\right)=u$, and $\phi\left(b_{2}\right)=u^{\prime}$. Again we have a repeated color (blue), and the embedding satisfies (5) and (6) (since both (11) and (12) do not hold).

To finish the embedding the rest of $L$, in both cases we do the following. We embed the remaining vertices in $L$ into a complete bipartite graph in the common neighborhoods in (5) and (6).

We embed $a_{3}$ into a vertex

$$
u \in\left(N_{G^{\prime \prime}}\left(\phi\left(b_{1}\right), C_{i}\right) \cap N_{G^{\prime \prime}}\left(\phi\left(b_{2}\right), C_{i}\right)\right) \backslash\left\{\phi\left(a_{1}\right), \phi\left(a_{2}\right)\right\}
$$

such that

$$
\left|N_{G^{\prime \prime}}\left(u, C_{j}\right) \cap N_{G^{\prime \prime}}\left(\phi\left(a_{1}\right), C_{j}\right) \cap N_{G^{\prime \prime}}\left(\phi\left(a_{2}\right), C_{j}\right)\right| \geq \frac{1}{5}\left(\frac{\alpha}{3}\right)^{3} N .
$$

(3) guarantees that such a $u$ exists.

Continuing this way, we always embed $a_{r}, 3<r \leq|A|$ into a vertex

$$
u \in\left(N_{G^{\prime \prime}}\left(\phi\left(b_{1}\right), C_{i}\right) \cap N_{G^{\prime \prime}}\left(\phi\left(b_{2}\right), C_{i}\right)\right) \backslash \cup_{s=1}^{r-1} \phi\left(a_{s}\right)
$$

such that

$$
\left|N_{G^{\prime \prime}}\left(u, C_{j}\right) \cap\left(\cap_{s=1}^{r-1} N_{G^{\prime \prime}}\left(\phi\left(a_{s}\right), C_{j}\right)\right)\right| \geq \frac{1}{5}\left(\frac{\alpha}{3}\right)^{r} N .
$$

(3) always guarantees that such a $u$ exists.

Finally, we embed $B \backslash\left\{b_{1}, b_{2}\right\}$ into vertices in

$$
\cap_{s=1}^{|A|} N_{G^{\prime \prime}}\left(\phi\left(a_{s}\right), C_{j}\right) \backslash\left\{\phi\left(b_{1}\right), \phi\left(b_{2}\right)\right\} .
$$

This is an embedding of $L$ into $G^{\prime \prime}$ (and thus into $G$ ) which contains a repeated color.

This completes the proof of Theorem 4.

\section{ACKNOWLEDGMENT}

Gábor N. Sárközy thanks M. Simonovits for helpful discussions on the topic. 


\section{REFERENCES}

[1] N. Alon, On a conjecture of Erdős, Simonovits and Sós concerning antiRamsey theorems, J Graph Theory 7 (1983), 91-94.

[2] N. Alon, H. Lefmann, V. Rödl, On an anti-Ramsey type result, in Sets, graphs and numbers, Budapest, 1991, Vol. 60 of Colloq Math Soc János Bolyai, North-Holland, Amsterdam, 9-22.

[3] M. Axenovich, T. Jiang, Anti-Ramsey numbers for small complete bipartite graphs, to appear in Ars Combinatoria. 73 (2004), 311-318.

[4] M. Axenovich, T. Jiang, and A. Kündgen, Bipartite anti-Ramsey numbers of cycles and path covers in bipartite graphs, J Graph Theory 47 (2004), 9-28.

[5] B. Bollobás, Extremal Graph Theory, Academic Press, London, 1978.

[6] S. A. Burr, P. Erdős, P. Frankl, R. L. Graham, and V. T. Sós, Further results on maximal antiramsey graphs, In Graph Theory, Combinatorics and Applications, Vol. I, Y. Alavi, A. Schwenk (Editors), John Wiley and Sons, New York, 1988, pp. 193-206.

[7] S. A. Burr, P. Erdős, R. L. Graham, and V. T. Sós, Maximal antiramsey graphs and the strong chromatic number, J Graph Theory 13 (1989), 263-282.

[8] P. Erdôs, M. Simonovits, V. T. Sós, Anti-Ramsey theorems, in Infinite and finite sets, (Colloq., Keszthely, 1973; dedicated to P. Erdős on his 60th birthday), Vol. II, North Holland, Amsterdam, Vol. 10 of Colloq Math Soc János Bolyai, 633-643.

[9] R. L. Graham, B. L. Rothschild, and J. H. Spencer, Ramsey Theory, 2nd edition, John Wiley and Sons, New York, 1990.

[10] J. Komlós and M. Simonovits, Szemerédi's Regularity Lemma and its applications in graph theory, In Combinatorics, Paul Erdős is Eighty, Vol. 2, D. Miklós, V. T. Sós, and T. Szőnyi, (Editors), Bolyai Society Mathematical Studies, Budapest, 1996, pp. 295-352.

[11] M. Simonovits, Some of my favorite Erdős theorems and related results, theories, In Paul Erdős and his mathematics, II, Budapest, Hungary, 2002, pp. 555-625.

[12] E. Szemerédi, Regular partitions of graphs, Colloques Internationaux C.N.R.S. No. 260-Problèmes Combinatoires et Théorie des Graphes, Orsay, 1976, pp. 399-401.

[13] T. Jiang, D. B. West, On the Erdôs-Simonovits-Sós conjecture about the antiRamsey number of a cycle, Comb, Prob Comput 12 (2003), 585-598. 